\title{
Langzeitprognose sehr unreifer Frühgeborener
}

\author{
Long-term Outcome for Very Preterm Neonates
}

L. Gortner

Bibliografie

DOI $10.1055 / \mathrm{s}-2008-1046761$

Klin Pädiatr 2008; 220: 55-56

(c) Georg Thieme Verlag KG

Stuttgart $\cdot$ New York

ISSN 0300-8630

Korrespondenzadresse

Prof. Dr. L. Gortner

Kliniken für Kinder- und

Jugendmedizin

Universitätsklinikum des

Saarlandes

66421 Homburg/Saar

ludwig.gortner@uks.eu
Durch die Erfolge der perinatalen Medizin wurde die Überlebensrate sehr unreifer Frühgeborener deutlich verbessert. Aktuell liegt die Rate des Überlebens für Kinder eines Gestationsalters unter 32 Wochen bei über $90 \%$, wobei im Vergleich verschiedener Europäischer Regionen in Deutschland exzellente Resultate erzielt werden [10].

Vor dem Hintergrund der angesprochenen Daten ergibt sich die Frage der Qualität des Überlebens sowie möglicher Behandlungsstrategien zu dessen weiterer Verbesserung. In der vorliegenden Ausgabe der Klinischen Pädiatrie wird über die Überlebensqualität sehr kleiner Frühgeborener im Erwachsenenalter berichtet [9], die eine Studie aus Graz mit Nachuntersuchungen im frühen Kindesalter ergänzt [8]. Die mittels Fragebogen untersuchte Kohorte der Jahrgänge 1979 bis 1986 wurde in einer Periode behandelt, in der die Therapie mit Surfactant noch nicht möglich und die Applikation antenataler Kortikosteroide noch nicht durchgängiger Standard waren. Wesentliche Resultate der Untersuchung stellen die hohe Rate von $27 \%$ mit hochschulberechtigendem und $37 \%$ mit mittlerem Schulabschluss dar. Dies ist in Übereinstimmung mit Resultaten aus anderen Staaten, wo Kohorten mit vergleichbaren neonatalen Charakteristika einbezogen wurden $[1,4]$.

Einschränkungen der aus den genannten Untersuchungen im Adoleszenten- bzw. Erwachsenenalter gezogenen Schlüsse sind die geringe Rate informativer Patienten, gemessen an der Grundgesamtheit und die mittels Fragebogen erhobenen Daten. Sehr viel valider wären Daten eines prospektiv angelegten Registers, in das die Ergebnisse flächendeckender Nachuntersuchungen eingespeist würden, z.B. auch unter Nutzung anderweitiger Routineuntersuchungen, wie im Rahmen des Schuleingangs. Hierdurch würden auch zuverlässig Kinder frühzeitig erkannt, die aufgrund von Defiziten in ihrer Entwicklung von einer Frühförderung profitieren würden.

Dieser Weg der möglichen Kooperation von wissenschaftlichen Arbeitsgruppen mit Institutionen des öffentlichen Gesundheitswesens liegt der Untersuchung der Frankfurter Gruppe zugrunde [7]. An einem kleineren Kollektiv wird belegt, dass Frühgeborene eines Gestationsalters von unter 30 Wochen einen Anteil von $0,9 \%$ an allen sonderpädagogischen Maßnahmen haben.
Dabei liegt der Anteil dieser Kinder an der Gesamtgeburtenrate in der gleichen Größenordnung von rund $1 \%$. Die typischen Einschränkungen liegen im Bereich der Lernbehinderungen, Seh- und Hörstörungen und motorischen Behinderungen.

Dies macht deutlich, dass ein Spektrum an Störungen nach früher Frühgeburtlichkeit möglich ist, was in der Nachsorge Berücksichtigung finden muss. Diese Überlegungen für die Nachsorge im Kontext mit der immer komplexer werdenden Therapie in der akuten postnatalen Phase machen deutlich, dass sehr unreife Frühgeborene nur in dafür ausgewiesenen hoch spezialisierten Zentren betreut werden sollten, die das gesamte Spektrum der Akuttherapie und der Nachsorge auf dem dafür nötigen hohen Niveau anbieten.

Die Implementierung der Resultate der Neonatalerhebungen und der nach Beschluss des Gemeinsamen Bundesausschusses obligaten Nachuntersuchungen in den ersten 2 Lebensjahren könnte hier die wesentliche Grundlage eines überregionalen Registers darstellen. Mit diesem könnten auch die Effekte neonataler Interventionen überprüft und weitere gezielte Nachsorgemodelle etabliert werden.

Resultate von Nachsorgeuntersuchungen können erhebliche politische Konsequenzen bedingen: Die Publikation der Populationsstudie mit Einschluss extrem unreifer Frühgeborener im Alter von 6 bis 8 Jahren - die EPICure-Studie [6] in Großbritannien - führte zu einer generellen Diskussion über die Therapie an der Grenze der Überlebensfähigkeit. Bei der Wertung der Rate mit einer Behinderung überlebender Kinder wurde oft übersehen, dass rund $90 \%$ eine Regelschule besuchten. Im gleichen Sinne sollte eine selektive Interpretation der Daten aus der Observationsstudie von Straßburg und Mitarbeitern [8] kritisch gesehen werden.

Der kardiopulmonalen Problematik Frühgeborener wendet sich eine weitere Arbeit der vorliegenden Ausgabe zu. Der pulmonale Gefäßwiderstand des Frühgeborenen als seltene, aber die Gasaustauschfunktion weiter beeinträchtigende Komplikation ist Gegenstand der Mitteilung von Eifinger und Mitarbeitern [2]. Neben der inhalativen Behandlung mit Stickstoffmonoxid (NO) ist die Applikation von Prostacyklin eine Alternative 
in der Behandlung dieser relevanten Komplikation. Bevor sicher fundierte Therapieempfehlungen geben werden können, sind jedoch klinisch kontrollierte Studie unter Zugrundelegung der zitierten Pilotdaten nötig [5]. Solche Studien sind nur durch eine multizentrische Kooperation mit sorgfältig definierten Protokollen möglich, bevor verlässliche Therapierichtlinien generiert werden können [3]. Eine kritische Anzahl von Studienteilnehmern ist nötig, um innerhalb vertretbarer Zeiträume valide Studienresultate zu generieren. Derartige prospektive und kontrollierte Studien sind an die Fortschreibung leistungsfähiger Zentren der Maximalversorgung geknüpft und könnten bei ihrer Durchführung durch ein klinisches Register bei der Planung und in der Durchführung in wesentlicher Weise unterstützt werden.

\section{Literatur}

1 Cooke $R W$. Health, lifestyle, and quality of life for young adults born very preterm. Arch Dis Child 2004; 89 (3): 201-206

2 Eifinger F, Sreeram N, Mehler K, Huenseler C, Kribs A, Roth B. Inhalatives Iloprost in der Therapie des pulmonalen Hypertonus bei sehr kleinen Frühgeborenen: Eine Pilotstudie. Klin Padiatr 2008; 220 (2): 65-68

3 Gortner L. Richtlinien für die Reanimation bei Früh- und Neugeborenen. Klin Pädiatr 2007; 219: 49
4 Hack M, Flannery DJ, Schluchter M, Cartar L, Borawski E, Klein N. Outcomes in young adulthood for very-low-birth-weight infants. N Engl J Med 2002; 346 (3): 149-157

5 Hoehn T, Krause MF, Bührer C. Meta-analysis of inhaled nitric oxide in premature infants: an update. Klin Padiatr 2006; 218 (2): 57-61

6 Marlow N, Wolke D, Bracewell MA, Samara M. EPICure Study Group. Neurologic and developmental disability at six years of age after extremely preterm birth. N Engl J Med 2005; 352 (1): 9-19

7 Schlösser RL, Krackardt B, Weber J, Lermann E. Welchen Anteil haben ehemalige Frühgeborene an der Prävalenz von sonderpädagogischen Maßnahmen bei Schulkindern einer deutschen Großstadt (Frankfurt am Main). Klin Pädiatr 2008; 220 (2): 56-59

8 Sommer C, Urlesberger B, Maurer-Fellbaum U, Kutschera J, Müller W. Neurodevelopmental outcome at 2 years in 23 to 26 weeks old gestation infants. Klin Padiatr 2007; 219 (1): 23-29

9 Straßburg HM, Leimer S, Platz A, Thomas W. Langzeitprognose sehr kleiner Frühgeborener in Deutschland. Klin Pädiatr 2008; 220 (2): 60-64

10 Zeitlin J, Draper ES, Kollée L, Milligan D, Boerch K, Agostino R, Gortner L, Reempts P Van, Chabernaud J-L, Gadzinowski J, Bréart G, Papiernik E, the MOSAIC research group. Differences in rates and short term outcome of live births before 32 weeks of gestation in Europe in 2003: results from the MOSAIC cohort. Pediatrics 2008; 121, in press 